\title{
Hydrodynamic properties of carbohydrate-coated dendrimers
}

\author{
Georges M. Pavlov ${ }^{a}$, Evgueniya V. Korneeva ${ }^{b}$, Kornelia Jumel ${ }^{c}$, Stephen E. Harding ${ }^{c}$, \\ E.W. Meijer ${ }^{\mathrm{d}}$, H.W.I. Peerlings ${ }^{\mathrm{d}}$, J. Fraser Stoddart ${ }^{\mathrm{e}}$, Sergey A. Nepogodiev ${ }^{\mathrm{f}, *}$ \\ a Institute of Physics, St Petersburg University, Ul'anovskaya ul. 1, 19890 St. Petersburg, Russia \\ ${ }^{\mathrm{b}}$ Institute of Macromolecular Compounds, Bolshoi Pr. 31, 199004 St. Petersburg, Russia \\ ${ }^{\mathrm{c}}$ NCMH unit, University of Nottingham, School of Biological Sciences, Sutton Bonington LE12 5RD, UK \\ ${ }^{\mathrm{d}}$ Laboratory of Macromolecular and Organic Chemistry, Eindhoven University of Technology, PO Box 513, NL-5600 MB Eindhoven, The Netherlands \\ ${ }^{\mathrm{e}}$ Department of Chemistry and Biochemistry, University of California, Los Angeles, 405 Hilgard Avenue, Los Angeles, CA 90095-1965, USA \\ ${ }^{\mathrm{f}}$ School of Chemistry, University of Birmingham, Edgbaston, Birmingham B15 2TT, UK
}

Received 6 May 1998; accepted 13 July 1998

\begin{abstract}
The hydrodynamic properties of poly(propylene imine) dendrimers, modified with outer-surface $\beta$-thiolactosyl residues ("lactodendrimers"') of generation number 1-5, have been investigated by velocity sedimentation, translational diffusion, and viscosity measurements in $0.165 \% \mathrm{NaCl}$ aqueous solution. It has been demonstrated that apparent molecular weights (from the Svedberg equation) $M_{\mathrm{sD}}$ of synthetic lactodendrimers, determined by sedimentation and diffusion experiments, are consistent with complete functionalization of initial poly(propylene imine) dendrimers with sugar residues. The values of $M_{\mathrm{sD}}$ range from 1900 to $45000 \mathrm{Da}$ for generation numbers $1-5$. Corresponding hydrodynamic ("equivalent Stokes") radii range from $\sim 10$ to $\sim 37 \AA$. Scaling relations of the conformation-dependent sedimentation coefficient, translational diffusion coefficient and intrinsic viscosity with molecular weight have been considered in detail and are all indicative of a spherical molecule with an inhomogeneous distribution of density (Mark-Houwink-Kuhn-Sakurada $a, b$ and $\varepsilon$, coefficients of $\sim 0.24, \sim 0.69$ and $\sim 0.31$, respectively). (C) 1999 Elsevier Science Ltd. All rights reserved.
\end{abstract}

Keywords: Carbohydrate; dendrimers; Hydrodynamic properties; Poly(propylene imine) dendrimers

\section{Introduction}

Dendritic macromolecules (i.e., the so-called "dendrimers" and "dendrons") are a relatively new class of polymeric compounds which can be distinguished from conventional polymers by their highly branched but symmetrical architectures and their relatively precise molecular structures (Tomalia et al., 1990; Tomalia and Durst, 1990; Issberner et al., 1995; Newkome et al., 1996; Fréchet and Hawker, 1996; Zeng and Zimmerman, 1997; Matthews et al., 1998). The application of "dendrimerization" to the construction of artificial molecules by means of chemical synthesis opens up new avenues for controlling such molecular parameters as size, shape, surface chemistry, and topology at the nanoscopic level. At the macroscopic level the potential benefits of well-defined dendritic structures are also clear. Some dendritic materials have been identified as nanoscopic host molecules (Hawker et al., 1993; Ottaviani et al., 1995; Newkome et al., 1991; Jansen

\footnotetext{
* Corresponding author
}

et al., 1994), dendritic catalysts (Bhyrappa et al., 1996; Brunner, 1995), liquid crystals (Kwon et al., 1995; Ponomarenko et al., 1996), gene vectors (Kukowska-Latallo et al., 1996), contrast agents for magnetic resonance imaging (Wiener et al., 1994) and as tools for immunoassay (Tam, 1996; Singh et al., 1996). Since the early 1980s the main emphasis in dendrimer research has been on their synthesis (Tomalia et al., 1990; Tomalia and Durst, 1990; Issberner et al., 1995; Newkome et al., 1996; Fréchet and Hawker, 1996; Zeng and Zimmerman, 1997; Matthews et al., 1998; Voit, 1995). This emphasis has resulted in the development of several different synthetic strategies. Collectively they permit the preparation of a wide range of molecular assemblies of varying complexity and high molecular weights. In recent years, the introduction of functional components into dendritic skeletons is an activity that has come to the forefront, giving rise to many novel functional dendrimers (Newkome et al., 1996; Fréchet, 1994; Peerlings and Meijer, 1997; Ramzi et al., 1998).

Theoretical treatments of the different possible dendritic types of polymers were first considered by Flory (1941a, b, 
c); Kuhn and Kuhn (1947); Tsvetkov (1951), and well before the first dendrimers were synthesised. Nowadays, the various theoretical aspects, concerning specific details of dendrimer architectures, are usually analyzed by numerical and computational methods (de Gennes and Hervet, 1983; Naylor et al., 1989; Lescanec and Muthukumar, 1990; Mansfield, 1993; Mansfield and Klushin, 1993; Chen and Cui, 1996; Murat and Grest, 1996; Boris and Rubinstein, 1996; La Ferla, 1997; Cai and Chen, 1997). The chemical structures of dendrimers are invariably investigated using spectroscopic methods (Wooley et al., 1997). By contrast, studies of the solution physical properties of dendrimers (Fréchet et al., 1994; Caminati et al., 1995; Shi and Rånby, 1996; Young et al., 1994; Gitsov and Fréchet, 1993; Stutz, 1995; Mourey et al., 1992; Aharoni et al., 1982; Fréchet and Hawker, 1995; Scherrenberg et al., 1998) have been somewhat limited. This situation is surprising, considering the potential that exists for producing such molecules with tailored chemical and physical properties.

Recently, the synthesis of a series of carbohydrate-coated dendrimers has been described in the literature (Ashton et al., 1997). These compounds represent members of the rapidly expanding family of neoglycoconjugates - the socalled glycodendrimers (Jayaraman et al., 1997; Aoi et al., 1997; Roy, 1996; Lindhorst, 1996). Five of the carbohydrate-coated dendrimers as described by Ashton et al. (1997) and identified by these workers in terms of their "generation number" $g(1-5)$ are the subject of the present hydrodynamic investigation. The carbohydrate residues of these compounds are located on the terminii of their dendritic chains and, on the basis of computer modelling, it has been suggested (Ashton et al., 1997) that, in the case

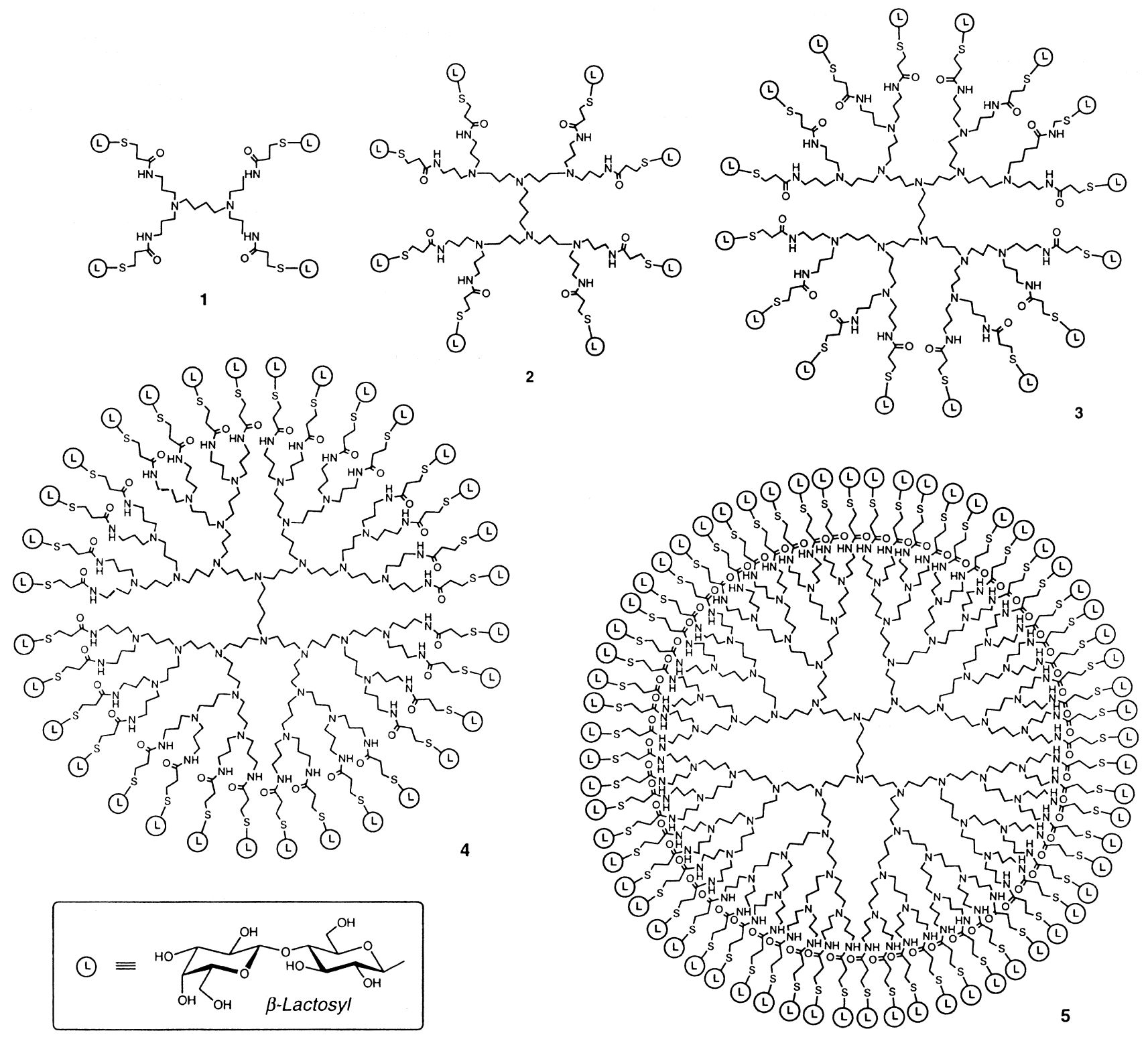

Fig. 1. Structural formulae of $\beta$-thiolactosyl modified poly(propylene imine) dendrimers (lactodendrimers, LDs) 1-5. 
of $g=5$, they form an outer layer shell covering the overall conformation of the macromolecule, which is presumably spheroidal. Important questions that need to be addressed include (i) what is the oligomeric/supramolecular state of these dendrimers in solution, i.e., is there any selfassociation/aggregation leading to supermolecules and, if so, what is the extent of aggregation? (ii) Does the overall shape of each assembly follow a general pattern, and if so, what is that pattern?

\section{Materials and methods}

\subsection{Samples and solvents}

The procedure for the synthesis of lactodendrimers (LDs) 1-5 (shown schematically in Fig. 1) was carried out as previously described (Ashton et al., 1997). They were prepared by modification of poly(propylene imine) dendrimers (de Brabander-van der Berg et al., 1996; de Brabander-van der Berg and Meijer, 1993) of "'generation number", $g=1$ to 5 by attaching spacer armed $\beta$-lactose derivatives. In the resulting compounds, lactose residues are attached to the ends of dendritic skeletons via stable amide bonds. Although the completeness of the substitution of terminal $\mathrm{NH}_{2}$ groups in parent dendrimers was confirmed for compounds $\mathbf{1}$ and $\mathbf{2}$ by matrix-assisted laser desorption/ionization time of flight mass spectrometry (MALDI-time of flight mass spectrometry), for the rest of the series, it has only been established by combustion analyses.

Samples were dried in vacuo before being measured for dry weight. For all hydrodynamic investigations, the samples were dispersed in $0.165 \%$ aqueous $\mathrm{NaCl}$. All hydrodynamic values were normalized to standard solvent conditions, namely the dynamic viscosity $\left(\eta_{0}=0.8931 \mathrm{cP}\right)$, and density $\left(\rho_{0}=0.9971 \mathrm{~g} / \mathrm{ml}\right)$ of water at a temperature of $25.0^{\circ} \mathrm{C}$.

\subsection{Translational diffusion}

The translational diffusion of dendrimers was studied by the so-called "classical method" (Tanford, 1961) involving the formation of a boundary between the solution and the solvent and recording the subsequent spreading of this interface with time. The studies were carried out using an in-house built diffusion cell as described in Tsvetkov et al. (1970) at a temperature of $26.0^{\circ} \mathrm{C}$, with the centre of the diffusion boundary being detected by a polarizing interferometer. Apparent translational diffusion coefficients $D$ at a loading concentration $c \sim 3 \mathrm{mg} / \mathrm{ml}$ were obtained from the dependence on time $t$ of the degree of interfacial dispersion $\sigma^{2}$ according to the equation (Tsvetkov et al., 1970)

$\sigma^{2}=\sigma_{0}^{2}+2 D t$

where $\sigma_{0}$ is the value of $\sigma$ at time $t=0$, and from the subsequent determination of the slope of plots of $2 \sigma^{2}$ versus time (Fig. 2).

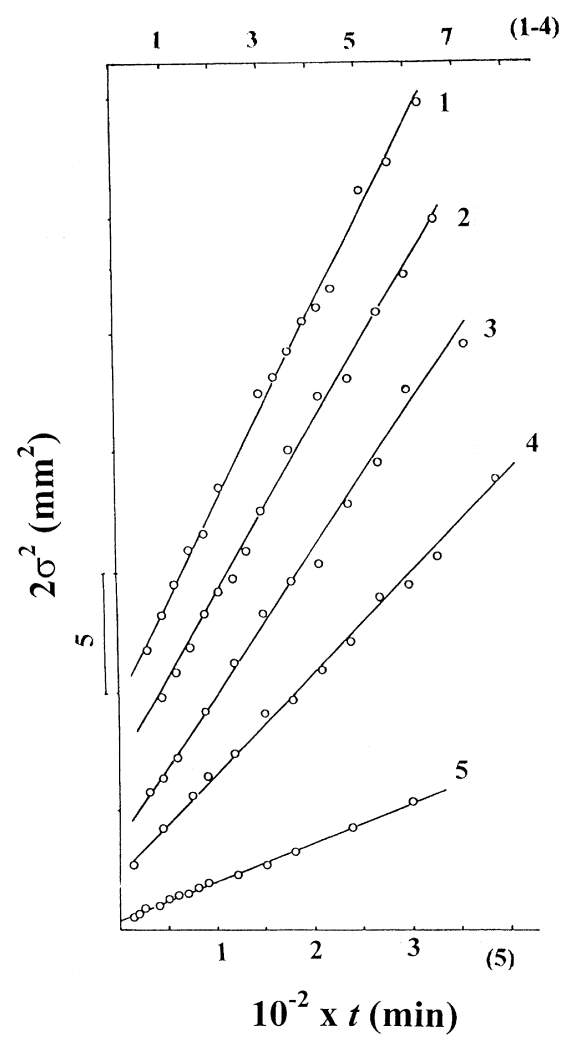

Fig. 2. Diffusivity of LDs. Time dependence of diffusion boundaries (second moment) for LDs $\mathbf{1 - 5}$ dispersed in $0.165 \% \mathrm{NaCl}$ solutions. For clarity, each curve is displaced along the ordinate axis by $2 \mathrm{~mm}^{2}$ relative to the previous curve, where $\sigma^{2}$ is the dispersion of the diffusion boundary calculated from the maximum ordinate and the area under the diffusion curve.

\subsection{Sedimentation velocity}

Sedimentation velocity studies were performed using both a MOM (Hungarian Optical Works, Budapest) 3180 analytical ultracentrifuge with a polarizing interferometer for recording the solution-solvent boundary and a Beckman (Palo Alto, USA) Model E analytical ultracentrifuge fitted with a Svennson-Philpott phase-plate/cylindrical lens Schlieren optical system, a rotor temperature indicator, a control (RTIC) device, and a mercury arc light source fitted with a green filter. Experiments were carried out in $12 \mathrm{~mm}$ optical path-length synthetic boundary cells and a rotor speed of $40000 \mathrm{rpm}$ at $20.0^{\circ} \mathrm{C}$. The apparent sedimentation coefficients $s$ for the same loading concentration as for the diffusion measurements (with a small correction for radial dilution) were calculated from conventional boundarymovement analysis by obtaining the slopes of plots fitted to the equation:

$s=\frac{d \ln r_{\mathrm{b}}}{d t} \omega^{-2}$

(Fig. 3) where $\omega$ is the angular velocity ( $\mathrm{rad} / \mathrm{s})$. 


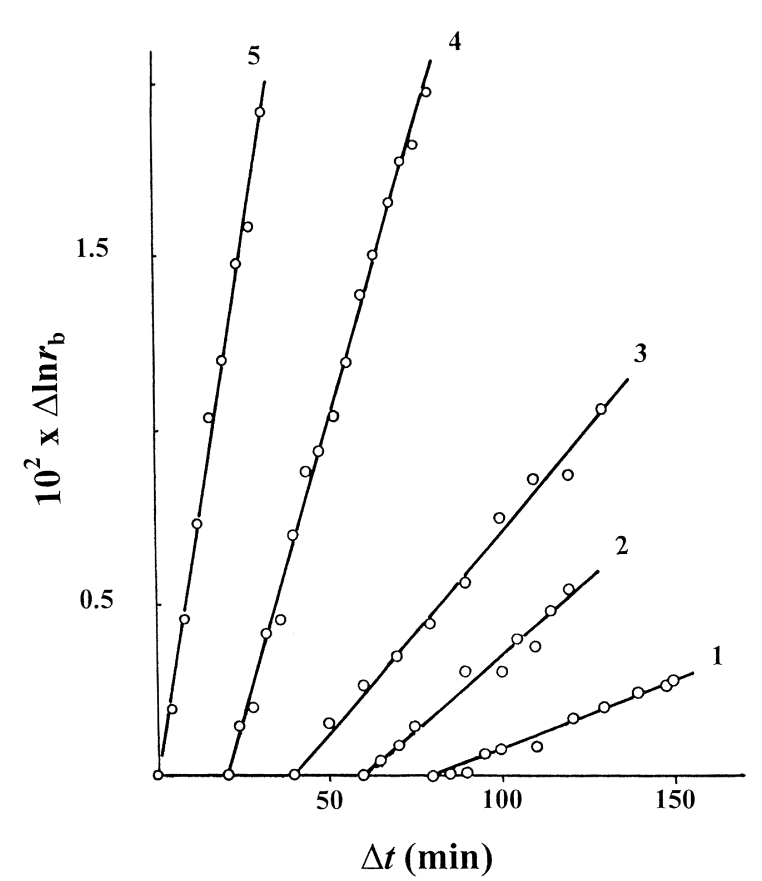

Fig. 3. Sedimentation velocity of LDs. Time dependence of sedimentation boundary movement for LDs $\mathbf{1 - 5}$ dispersed in $0.165 \% \mathrm{NaCl}$ solutions. Rotor speeds: $40000 \mathrm{rpm}$ for LDs 1-3 and $47660 \mathrm{rpm}$ for LDs 4 and $\mathbf{5}$. For clarity, each curve is displaced along the $\Delta t$ axis by $20 \mathrm{~min}$ relative to the previous curve.

\subsection{Intrinsic viscosity}

Flow times were measured using an Ostwald capillary viscometer with solution concentrations ranging from $2 \mathrm{mg} / \mathrm{ml}$ to $14 \mathrm{mg} / \mathrm{ml}$. All measurements were performed at $25.00^{\circ} \mathrm{C}$ with temperature monitored by an accurately calibrated platinum resistance thermometer. Intrinsic viscosities $[\eta]$ were determined by calculating relative viscosities, $\eta_{\text {rel }}$, converting to specific viscosity $\left(\eta_{\mathrm{sp}}=\eta_{\text {rel }}-1\right)$ and by extrapolating values of $\eta_{\mathrm{sp}} / c$ and $\ln \left(\eta_{\text {rel }} / c\right)$ to zero concentration using Huggins (1942) and Kraemer (1938) plots based on the respective relationships

$\frac{\eta_{\mathrm{sp}}}{c}=[\eta]+K_{\mathrm{H}}[\eta]^{2} c$

where $K_{\mathrm{H}}$ is the Huggins parameter and

$\frac{\ln \left(\eta_{\mathrm{rel}}\right)}{c}=[\eta]-K_{\mathrm{K}}[\eta]^{2} c$

where $K_{\mathrm{K}}$ is the Kraemer parameter (see e.g., Harding (1997)).

\subsection{Partial specific volumes}

Density measurements, $\rho$, were obtained using an Anton Paar (Graz, Austria) mechanical oscillator type of density meter (Model DMAC02C) and partial specific volumes, $\overline{\mathrm{v}}$, obtained from density increment measurement, $d \rho / d c$, according to the procedure of Kratky et al. (1973). All measurements were performed at $25.00^{\circ} \mathrm{C}$ with temperature monitored by an accurately calibrated platinum resistance thermometer.

\section{Results and discussion}

\subsection{Solubility}

The lactodendrimers 1-5 were all readily soluble in pure water. However, during the course of the translational diffusion experiments, it was revealed that, in the absence of a low molecular weight electrolyte, the diffusion profiles had pronounced asymmetries which did not disappear with time. This effect can be attributed to the polycationic nature of the poly(propylene imine) dendritic cores: further addition of a low molecular weight electrolyte, namely $\mathrm{NaCl}$, to the solutions made it possible to obtain symmetric diffusion curves. Thus, all subsequent hydrodynamic experiments were carried out in aqueous $\mathrm{NaCl}$ solution.

\subsection{Molecular weights of lactodendrimers}

Apparent "sedimentation-diffusion" molecular weights $M_{\mathrm{sD}}$ of LDs 1-5 were evaluated from the combination of the (apparent) sedimentation coefficient, $s$, with the (apparent) translational diffusion coefficient, $D$, according to the classical Svedberg equation (Svedberg and Pederson, 1940);

$M_{\mathrm{SD}}=\frac{R T}{\left(1-\overline{\mathrm{v}} \rho_{0}\right)} \times \frac{s}{D}$

where $R$ is the gas constant, $T$ is the temperature in $\mathrm{K}$ (all measurements were normalised to $25.0^{\circ} \mathrm{C}$ as indicated above), $\overline{\mathrm{v}}$ is the partial specific volume of the polymer and $\rho_{0}$ the solvent density. The correction for non-ideality was assumed small (and will effectively cancel anyway if the dependencies are similar for both the $s$ and $D$ values). Buoyancy factors $\left(1-\overline{\mathrm{v}} \rho_{0}\right)$ have been derived from the $\overline{\mathrm{v}}$ and $\rho_{0}$ values for LDs $\mathbf{1}, \mathbf{4}$ and $\mathbf{5}$ (Table 1) and were, respectively, $0.292,0.315$, and 0.332 . Assuming a monotonic dependence of $\bar{v}$ on $M_{\mathrm{sD}}$, the corresponding values of $\left(1-\overline{\mathrm{v}} \rho_{0}\right)$ for LDs 2 and $\mathbf{3}$ have been estimated as $(0.296 \pm 0.040)$ and $(0.304 \pm$ $0.040)$. The $D, s$ and corresponding $M_{\mathrm{sD}}$ values for the LDs are all listed in Table 1.

The experimental molecular weight values for the low generation LDs $(g=1-3)$ are in a good agreement with the monomer molecular weights $M_{1}$ (Table 1 ) corresponding to the chemical formulae. By contrast, the values of $M_{\mathrm{sD}}$ for the fourth- and fifth-generation LDs ( $g=4$ and 5) are $\sim 40 \%$ higher than corresponding calculated values for the monomer $M_{1}$. The incorporation of $\beta$-lactose residues into the initial poly(propylene imine) dendrimers can be less but not more than the theoretically possible number (32 residues in the case of $\mathbf{4}$ and 64 residues in the case of $\mathbf{5}$ ), so the $M_{1}$ values of Table 1 are the theoretical maxima. It follows that the only plausible explanation is that the $M_{\mathrm{sD}}$ values represent a partial self-association of these lactodendrimers. 
Table 1

Hydrodynamic and molecular characteristics of lactodendrimers $\mathbf{1 - 5 ^ { a }}$

\begin{tabular}{|c|c|c|c|c|c|c|c|c|}
\hline Lactodendrimer & {$[\eta](\mathrm{ml} / \mathrm{g})$} & $10^{7} \times D\left(\mathrm{~cm}^{2} / \mathrm{s}\right) s$ & (S) & $M_{\mathrm{sD}}(\mathrm{g} / \mathrm{mol})$ & $M_{1}^{\mathrm{b}}(\mathrm{g} / \mathrm{mol})$ & $10^{8} \times r_{\mathrm{f}}(\mathrm{cm})$ & $10^{8} \times r_{\eta}(\mathrm{cm})$ & $A_{0}$ \\
\hline 1 & 3.2 & 17.7 & 0.40 & 1900 & 1966 & 13.8 & 9.9 & 2.09 \\
\hline 2 & 3.9 & 14.6 & 0.75 & 4300 & 4073 & 16.7 & 13.9 & 2.43 \\
\hline 3 & 4.9 & 12.25 & 1.2 & 8000 & 8285 & 19.9 & 18.4 & 2.73 \\
\hline 4 & 5.6 & 8.5 & 2.6 & 24000 & 16711 & 28.7 & 27.7 & 2.81 \\
\hline 5 & 7.0 & 6.75 & 4.2 & 46500 & 33562 & 36.2 & 37.2 & 3.00 \\
\hline
\end{tabular}

${ }^{\mathrm{a}}$ Experimental data are obtained in $0.165 \%$ aqueous $\mathrm{NaCl}$ at $25.0^{\circ} \mathrm{C}$.

${ }^{\mathrm{b}}$ Molecular weight from chemical formula.

\section{Molecular size and hydrodynamic radii of lactodendrimers}

The molecular sizes of molecules of $\mathbf{1 - 5}$ have been estimated by calculating the radii of their hydrodynamically equivalent (and rigid) spheres $r$. These values have been obtained from both the translation frictional coefficient, giving $r_{\mathrm{f}}$, and from the intrinsic viscosity, giving $r_{\eta}$. The radii $r_{\mathrm{f}}$ (Table 1) for $\mathbf{1 - 5}$ have been calculated on the basis of the data on diffusion coefficients $D$ according to the Stokes-Einstein equation (see Tanford (1961))

$f=6 \pi \eta_{0} r_{\mathrm{f}}$

where $f$ is the translational frictional coefficient whose relation to $D$ is given by

$D=\frac{k T}{f}$

where $k$ is the Boltzmann constant. Conversely, $r_{\eta}$ values have been obtained from the viscometry data, using the Einstein equation which can be given as (Tanford, 1961; Tsvetkov et al., 1970)

$[\eta]=2.5 N_{\mathrm{A}}(V / M)$

where $N_{\mathrm{A}}$ is Avogadro's number, $V$ is the molecular volume (including solvation), and $M$ is the molecular weight. From this equation, the hydrodynamic radius $r_{\eta}$ can be calculated as

$r_{\eta}=\left[\frac{3 M[\eta]}{10 \pi N_{\mathrm{A}}}\right]^{\frac{1}{3}}$

There are some differences between the values of $r_{\mathrm{f}}$ and $r_{\eta}$ listed in Table 1: these differences are evident for low generation numbers $g$ and tend to vanish at high $g$. Within the range of $r_{\mathrm{f}}$ and $r_{\eta}$ values investigated, the correlation between the two radii may be represented by an empirical relation:

$r_{\eta} \sim 1.219 r_{\mathrm{f}}-6.9$

or in the form of the power function

$r_{\eta} \sim 2.37 r_{\mathrm{f}}^{0.75}$

\subsection{Distribution of saccharide residues}

Molecular dynamic simulations of the structure of the lactodendrimer 5 (Ashton et al., 1997), as well as results from molecular modelling studies on $N$ - $t$-bytuloxycarbonyl-L-phenylalanyl (or $N$ - $t$-BOC-L-phenylalanyl) modified poly(propylene imine) dendrimer of the fifth generation ( $g=5$ ) (Jansen et al., 1995), have indicated that bulky saccharide residues are located on the outer surfaces of the relatively rigid poly(propylene imine) cores and are not evenly distributed throughout the molecular volume of the LDs. It is worth noting also that the carbohydrate part of each lactodendrimer makes up about $80 \%$ of its mass. Taking into account these features of the lactodendrimers composition, a spherical "hollow" shell, rather than a homogeneous sphere, would, we believe, appear to be a more appropriate hydrodynamic model. The validity of either of these models can be examined by using the intrinsic viscosity data for LDs $\mathbf{1 - 5}$. Because of the fact that the LDs apparently represent a series of homologous compounds, the Mark-Houwink-Kuhn-Sakurada (MHKS) relation (see, e.g., Bohdanecky and Kovar, 1982; Harding, 1997)

$[\eta]=K^{\prime} M^{a}$

could be applied to probe their structure via the exponent $a$. If the LDs 1-5 are considered as rigid impermeable homogeneous spheres, then the variation of their intrinsic viscosity values versus molecular weight $M$ over the range of LDs studied (Fig. 4) should give a value for the scaling

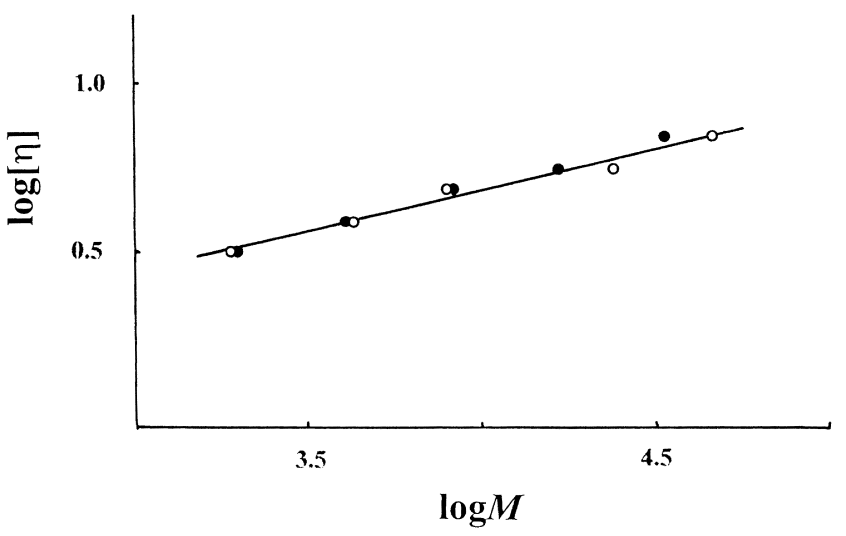

Fig. 4. Double-logarithmic MHKS (Mark-Houwink-Kuhn-Sakurada) plot of $[\eta]$ versus molecular weights evaluated, respectively, from the Svedberg equation, $M_{\mathrm{sD}}$ (open symbols) and monomer values calculated from the chemical formula $M_{1}$ (filled symbols) for LDs $\mathbf{1}-\mathbf{5}$. 


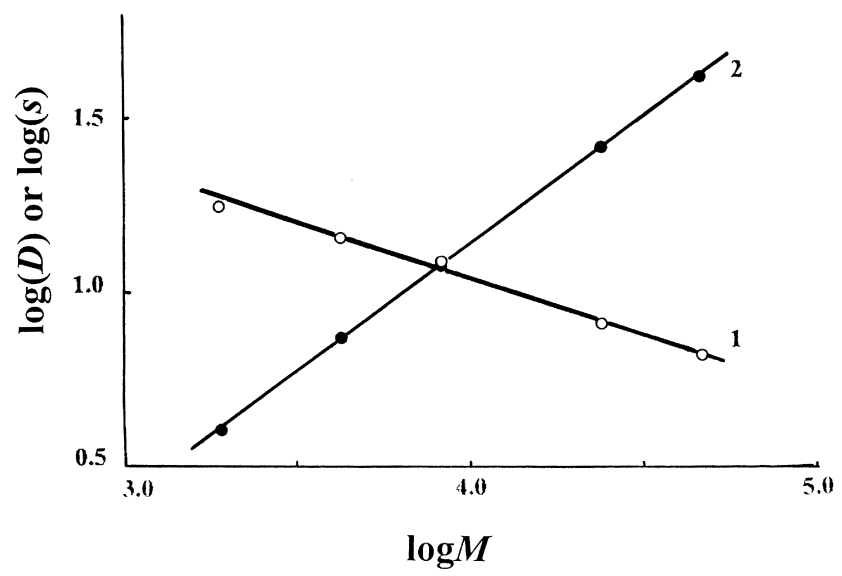

Fig. 5. Double-logarithmic MHKS (Mark-Houwink-Kuhn-Sakurada) plot of sedimentation coefficient, $s$ (open symbols) and translational diffusion coefficient $D$ (closed symbols) versus molecular weights, $M_{\mathrm{sD}}$, evaluated from the Svedberg equation for LDs 1-5.

index (exponent $a$ above) of $\sim 0$ (Bohdanecky and Kovar, 1982; Harding, 1997). In fact, the data gave a value for $a$ of $(0.235 \pm 0.021)$ with a linear correlation parameter $R=$ 0.9885. In Fig. 4, the viscosity data are also shown as a function of the monomer molecular weight, $M_{1}$ (calculated from the structural formulae). Since the form of the dependence of $[\eta]$ on $M_{1}$ is virtually the same as from the experimental $M_{\mathrm{sD}}$, with a slightly higher value for $a=0.272 \pm$ $0.011(R=0.9977)$, the homogeneous sphere model can be safely ruled out. In fact, both values for the scaling index $a$ are intermediary between those expected for a homogeneous sphere (0) and a random coil (0.5-0.8). Translational frictional data are also well described by similar scaling relationships (Fig. 5): $s \sim M^{\mathrm{b}}$ and $D \sim M^{-\varepsilon}$, where, $\varepsilon=$ $1-b$. We observe experimentally $b=(0.692 \pm 0.015)$ and, $\varepsilon=(0.305 \pm 0.013)$ for the lactodendrimers. These values, like $a$, again deviate from the expected values for a homogeneous sphere (0.667 and 0.333, respectively).

It is also worth noting that, for a homologous series of polymers, the MHKS $a$ and $\varepsilon$ exponents should be simply related by the relation (Tsvetkov et al., 1970)

$\varepsilon=(1 / 3)(a+1)$

Our data suggests that Eq. (11) does not hold exactly (although the deviation is relatively small), indicating that the polymers do not form a perfect homologous series. When passing from one generation of lactodendrimer to another, changes in the hydrodynamic interactions inside the volume shell occupied by a molecule are also possible. In other words, the degree of draining could depend on the dendrimer generation. If this situation pertains then it may be reflected in the values of the useful hydrodynamic “invariant" $A_{0} \equiv k \beta$ (Tsvetkov et al., 1970, 1984), where $\beta$ is the Flory-Mandelkern parameter (Mandelkern and Flory, 1952). The value for $A_{0}$ can be calculated from the experimental data for $[\eta], D$, and $s$ using, for example, the equation (Pavlov et al., 1990)

$A_{0}=\left(R \times[D]^{2} \times[s] \times[\eta]\right)^{1 / 3}$

where $[D] \equiv D \eta_{0} / T,[s] \equiv s \eta_{0} /\left(1-v \rho_{0}\right)$. The calculated values of $A_{0}$ for the series of LDs 1-5 (Table 1) show a clear monotonic increase in $A_{0}$ with increasing generation number, $g$. For higher generation numbers, the $A_{0}$ values are close to those that can be obtained from the StokesEinstein hydrodynamic coefficients for impermeable spheres $\left(A_{0 \text { sph }}=2.914 \times 10^{-10} \mathrm{erg} \mathrm{mol}^{1 / 3} / \mathrm{K}\right)$

$A_{0} \equiv k P_{0}^{-1} \Phi_{0}^{1 / 3}$

$P_{0}=6 \pi$

$\Phi_{0}=2.5 N_{\mathrm{A}} \frac{4}{3} \pi=\frac{\pi N_{\mathrm{A}}}{30}$

with $[\eta]$ in $\mathrm{ml} / \mathrm{g}$ (see Tsvetkov et al., 1970, 1984). For linear chain molecules, $A_{0}$ is virtually constant when $L / A>10$, where $A$ is the Kuhn segment length. (Tsvetkov et al., 1984). A monotonic change in $A_{0}$ can be predicted from the Debye and Bueche (1948) treatment for describing the translational and rotational motion of a homogeneous draining sphere. In this case $A_{0}$ depends on the hydrodynamic permeability of the molecules. The theory describing the hydrodynamic behaviour of a solid cylinder in the absence of volume interactions between its parts ( $\theta$-conditions) assumes that $A_{0}$ decreases with decreasing $L / A$ at $L / A<1$ (Tsvetkov et al., 1984; Yamakawa and Fujii, 1973, 1974). The change in $A_{0}$ observed by us here implies that both $P_{0}$ and $\Phi_{0}$ (or at least one of them) are not constant in the series of structures being investigated. If the fundamental principle of equivalent molecular dimensions during translational and rotational molecular motion is observed (Tsvetkov et al., 1970), this fact implies that $\Phi_{0}<\pi N_{\mathrm{A}} / 30$ and/or $P_{0}>6 \pi$ for the lowest generation numbers $g$. Non-constancy of the hydrodynamic coefficients $\Phi_{0}$ and $P_{0}$ does not allow us, however, to infer on the fractal dimensions of the lactodendrimers under investigation from the scaling parameters $a, b$ or $\varepsilon$. Another complication that restricts interpretation of the data is (as is apparent from Table 1) the clear evidence for selfassociation for the two higher generation numbers of glycodendrimer (4 and 5) which stretches the assumption of homologous series behaviour would deviate the behaviour away from a homologous series. Any further attempts at interpretation of the data awaits (i) new experimental data for other series of glycodendrimer, and (ii) development of hydrodynamic theory of super-branched molecules.

\section{Conclusions}

This is the first time, as far as we are aware, that the sedimentation, diffusion and viscometric characteristics of lactodendrimers (LDs) have been reported collectively. Our studies on the lactodendrimer generations (1-5) have shown 
that the measured values of the molecular weight are not at variance with the concept of complete substitution of end groups in the parent dendropropylenimine chains by lactose residues. Viscometry has shown clearly that LD molecules have properties commensurate with an assembly intermediate between an impermeable solid sphere and a spherical shell configuration. In contrast to the relative insensitivity of the intrinsic viscosity of the LDs to molecular weight (the MHKS $a$ coefficient is only $\sim 0.24$ ), the translational friction based parameters (sedimentation coefficient $s$ and diffusion coefficient $D$ ) are more sensitive, with MHKS (scaling) coefficients of $b \sim 0.69$ and, $\varepsilon \sim 0.31$. All these data are consistent with a spherical shell structure for the lactodendrimers.

\section{Acknowledgements}

This research was supported by the Russian Foundation for Fundamental Investigations (RFFI) (Grant No 96-0333847a) in the Russian Federation, the Biomolecular Sciences Committee of the Engineering and Physical Sciences Research Council (EPSRC) and Biotechnology and Biomedical Sciences Research Council (BBSRC) in the UK, the Underwood Foundation of the BBSRC, The Netherlands Foundation for Chemical Research (SON), and by the Netherlands Organization for Scientific Research (NWO) in the Netherlands. DSM is gratefully acknowledged for providing us with the poly(propylene imine) dendrimers.

\section{References}

Aharoni, S.M., Crosby, C.R. III, \& Walsh, E.K. (1982). Macromolecules, 15, 1093-1098.

Aoi, K., Itoh, K., \& Okada, M. (1997). Macromolecules, 30, 8071, 8074.

Ashton, P.R., Boyd, S.E., Brown, C.L., Nepogodiev, S.A., Meijer, E.W., Peerlings, H.W.I., \& Stoddart, J.F. (1997). Chem. Eur. J., 3, 974-984.

Bhyrappa, P., Young, J.K., Moore, J.S., \& Suslick, K.S. (1996). J. Am. Chem. Soc., 118, 5708-5711.

Bohdanecky, M., \& Kovar, J. (1982). Viscosity of polymer solutions. Amsterdam: Elsevier.

Boris, D., \& Rubinstein, M. (1996). Macromolecules, 29, 7251-7260.

Brunner, H. (1995). J. Organometallic Chem., 500, 39-46.

Cai, C.Z., \& Chen, Z.U. (1997). Macromolecules, 30, 5104-5117.

Caminati, G., Ottaviani, M.F., Gopidas, K., Jockusch, S., Turro, N.J., \& Tomalia, D.A. (1995). Proc. Am. Chem. Soc. Division of Polymeric Materials: Science and Engineering, 73, 80-81.

Chen, Z.Yu., \& Cui, S.-M. (1996). Macromolecules, 29, 7943-7952.

de Brabander-van der Berg, E.M.M., Brackman, J., Mure-mak, M., de Man, H., Hogeweg, M., Keulen, J., Scherrenberg, R., Coussens, B., Mengerink, Y., \& van der Wal, S. (1996). Macromol. Symp., 102, 9-17. de Brabander-van der Berg, E.M.M., \& Meijer, E.W. (1993). Angew. Chem. Int. Ed. Engl., 32, 1308-1311.

de Gennes, P.G., \& Hervet, H. (1983). J. Phys. Lett. Fr., 44, L351-L360.

Debye, P., \& Bueche, A.M. (1948). J. Chem. Phys., 16, 573-579.

Flory, P.J. (1941). J. Am. Chem. Soc., 63, 3083-3090.
Flory, P.J. (1941). J. Am. Chem. Soc., 63, 3091-3095.

Flory, P.J. (1941). J. Am. Chem. Soc., 63, 3096-3100.

Fréchet, J.M.J. (1994). Science, 263, 1710-1715.

Fréchet, J.M.J., \& Hawker, C.J. (1995). React. Func. Polym., 26, 127-136.

Fréchet, J.M.J., \& Hawker, C.J. (1996). In S.L. Aggarwal \& S. Russo (Eds.), Comprehensive polymer science (pp. 140-201).

Fréchet, J.M.J., Hawker, C.J., \& Wooley, K.L.J. (1994). Macromol. Sci. Pure Appl. Chem., A31, 1627-1645.

Gitsov, I., \& Fréchet, J.M.J. (1993). Maromolecules, 26, 6536-6546.

Harding, S.E. (1997). Prog. Biophys. Mol. Biol., 68, 207-262.

Hawker, C.J., Wooley, K.L., \& Fréchet, J.M.J. (1993). J. Chem. Soc., Perkin Trans., 1, 1287-1297.

Huggins, M.L. (1942). J. Am. Chem. Soc., 64, 2716-2718.

Issberner, J., Moors, R., \& Vögtle, F. (1995). Angew. Chem. Int. Ed. Engl., $33,2413-2420$.

Jansen, J.F.G.A., de Brabander-van den Berg, E.M.M., \& Meijer, E.W. (1994). Science, 266, 1226-1229.

Jansen, J.F.G.A., Peerlings, H.W.I., de Brabander-van den Berg, E.M.M., \& Meijer, E.W. (1995). Angew. Chem. Int. Ed. Engl., 34, 1206-1209.

Jayaraman, N., Nepogodiev, S.A., \& Stoddart, J.F. (1997). Chem. Eur. J., 3, 1193-1199.

Kraemer, E.O. (1938). Ind. Eng. Chem., 30, 1200.

Kratky, O., Leopold, H., \& Stabinger, H. (1973). Methods in Enzymology, $27,98-110$.

Kuhn, W., \& Kuhn, H. (1947). Helv. Chim. Acta., 30, 1233-1256.

Kukowska-Latallo, J.F., Bielinska, A.U., Johnson, J., Spindler, R., Tomalia, D.A., \& Baker, J.R. Jr (1996). Proc. Natl. Acad. Sci. USA, 93, 48974902.

Kwon, Y.K., Chvalun, S.N., Blackwell, J., Percec, V., \& Heck, J.A. (1995). Macromolecules, 28, 1552-1558.

La Ferla, R.J. (1997). Chem. Phys., 106, 688-700.

Lescanec, R.L., \& Muthukumar, M. (1990). Macromolecules, 23, 22802288.

Lindhorst, T.K. (1996). Nachr. Chem. Tech. Lab., 44, 1073-1079.

Mandelkern, L., \& Flory, P.J. (1952). J. Chem. Phys., 20, 212-214.

Mansfield, M.L. (1993). Macromolecules, 26, 3811-3814.

Mansfield, M.L., \& Klushin, L.I. (1993). Macromolecules, 26, 4262-4268.

Matthews, O.A., Shipway, A.N., \& Stoddart, J.F. (1998). Prog. Polym. Sci., $23,1-56$.

Mourey, T.H., Turner, S.R., Rubinstein, M., Fréchet, J.M.J., Hawker, C.J., \& Wooley, K.L. (1992). Macromolecules, 25, 2401-2406.

Murat, M., \& Grest, G.S. (1996). Macromolecules, 29, 1278-1285.

Naylor, A.M., Goddard, W.A. III, Liefer, G.E., \& Tomalia, D.A. (1989). J. Am. Chem. Soc., 111, 2339-2341.

Newkome, G.R., Moorefield, C.N., Baker, G.R., Saunders, M.J., \& Grossman, S.H. (1991). Angew. Chem. Int. Ed. Engl., 30, 1178-1180.

Newkome, G.R., Moorefield, C.N., \& Vögtle, F. (1996). Dendritic macromolecules. Weinheim: VCH.

Ottaviani, M.F., Cossu, E., Turro, N.J., \& Tomalia, D.A. (1995). J. Am. Chem. Soc., 117, 4387-4398.

Pavlov, G.M., Panarin, E.E., Korneeva, E.V., Kurochkin, C.V., Baikov, V.E., \& Ushakova, V.N. (1990). Makromol. Chem., 191, 2889-2899.

Peerlings, H.W.I., \& Meijer, E.W. (1997). Chem. Eur. J., 3, 1563.

Ponomarenko, S.A., Rebrov, E.A., Bobrovsky, A.Y., Boiko, N.I., Muzafarov, A.M., \& Shibaev, V.P. (1996). Liquid Cryst., 21, 1-12.

Ramzi, A., Scherrenberg, R., Brackman, J., Joosten, J., \& Mortensen, K. (1998). Macromolecules, 31, 1621.

Roy, R. (1996). Polymer News, 21, 226-232.

Scherrenberg, R., Coussens, B., van Vliet, P., Edouard, G., Brackman, J., \& de Brabender, E. (1998). Macromolecules, 31, 456-461.

Shi, W., \& Rånby, B. (1996). J. Appl. Polym. Sci., 59, 1937-1944.

Singh, P., Moll, F. III, Lin, S.H., \& Ferzli, C. (1996). Clin. Chem., 42, $1567-1569$.

Stutz, H. (1995). J. Polym. Sci., 33, 333-340.

Svedberg, T., \& Pedersen, K.O. (1940). The ultracentrifuge. Oxford, UK, Oxford University Press.

Tam, J.P. (1996). J. Immunol. Methods, 196, 17-32. 
Tanford, C. (1961). Physical chemistry of macromolecules. New York: Wiley. Tomalia, D.A., Naylor, A.M., \& Goddard, W.A. III (1990). Angew. Chem. Int. Ed. Engl., 29, 138-175.

Tomalia, D.A., \& Durst, H.D. (1990). Top. Curr. Chem., 165, 193-313.

Tsvetkov, V.N. (1951). Dokl. Akad. Nauk SSSR, 78, 1123-1126.

Tsvetkov, V.N., Eskin, V.E., \& Frenkel, S.Ya. (1970). Structure of macromolecules in solutions. London: Butterworths.

Tsvetkov, V.N., Lavrenko, P.N., \& Bushin, S.V. (1984). J. Polym. Sci., Pol. Chem. Ed., 22, 3447-3486.

Voit, B.I. (1995). Acta Polym., 46, 87-99.
Wiener, E.C., Brechbiel, M.W., Brothers, H., Magin, R.L., Gansow, O.A., Tomalia, D.A., \& Lauterbur, P.C. (1994). Magn. Reson. Med., $31,1-8$.

Wooley, K.L., Klug, C.L., Tasaki, K., \& Schaefer, J. (1997). J. Am. Chem. Soc., 119, 53-58.

Yamakawa, H., \& Fujii, M. (1973). Macromolecules, 6, 407-415.

Yamakawa, H., \& Fujii, M. (1974). Macromolecules, 7, 128-135.

Young, J.K., Baker, G.R., Newkome, G.R., Morris, K.F., \& Johnson, C.S. Jr (1994). Macromolecules, 27, 3464-3471.

Zeng, F., \& Zimmerman, S.C. (1997). Chem. Rev., 97, 1681-1712. 